\section{On the estimation of absorption of subcutaneous injected insulin from plasma concentrations using mathematical models}

Dear Sir,

Knowledge of the absorption rate of insulin after SC injection would have considerable value in facilitating quantitative investigation of degradation, bioavailability and other attributes of insulin administered by this route. However, despite the conceptual simplicity of the problem, its reliable solution is critically dependent upon both the design of the experimental protocol and the modelling method employed for data analysis.

A recent paper in Diabetologia has addressed this problem in the dog [1]. We would like to comment on some basic aspects of the experimental protocol and the modelling method used. As we are addressing a similar problem in man [2,3], we also include in support some of our current findings.

1. Two fundamental assumptions made by the authors concern IV insulin kinetics. The first is that the response to an IV insulin injection, and thus plasma insulin clearance rate, are independent of the glucose level. This is important because computation from plasma concentrations of the total amount of insulin appearing in plasma after SC injection requires knowledge of the plasma clearance rate, which must be constant during the experiment. The assumption that it is constant may not be justified: we have found insulin clearance to be about $15 \%$ greater in hypoglycaemia than in normoglycaemia. Thus the estimate of total insulin appearing in plasma will be affected by a serious error if clearance varies by a considerable extent during the experiment.

The second assumption is that rate of appearance of $\mathrm{SC}$ insulin may be computed from IV and SC insulin responses obtained on different days. As the response to an IV insulin injection seems to show considerable day-to-day variation in the same individual (about 25\% for the clearance estimate in normal human subjects, in our experience), incorrect results may be obtained in individual studies. Averaging may in part compensate for this kind of error, but at the expense of a larger standard deviation.

2. The modelling method plays an essential role in the study of Fischer et al. [1]. In general, when mathematical modelling is employed and is relevant for the intended purpose (as it is here), assessment of its validity [4] should receive at least as much attention as other aspects, such as patient selection and analytical methods. To predict from plasma concentrations the time course of the rate of insulin appearance after SC injection [1] requires mathematical modelling methods of some complexity. It is important that such methods should be well documented and quantitatively validated [4]. Fischer et al. [1] use three mathematical models with practically no evidence or discussion of their appropriateness:

a) A linear, one-compartment model for insulin kinetics after IV injection is used but no details to support its validity are given; no details are given on the insulin measurement errors nor on the quantitative criteria used in the model selection process, such as goodness-of-fit, residual errors and coefficient of variation of estimated parameters. Details on the modelling process are particularly important here since there is no reasonable agreement as to which model is the most appropriate for describing insulin kinetics. This problem is completely ig- nored, even in the reference list, where relevant contributions on the kinetics of insulin $[5,6]$ are neglected. Our current results agree with Tranberg and Dencker [6] in suggesting that the choice of the model is not straightforward; in some cases one exponential is satisfactory, while in others two exponentials are necessary. The consequences of using an incorrect model can be exemplified at a number of levels; for instance, if we compare in our normal population the clearance values computed from a crude, one-exponential model with those obtained after more careful model selection, we find a statistically significant difference of about $15 \%$.

b) A two-compartment linear model is implicitly assumed to be applicable to the kinetics of SC insulin absorption. Again, as above, no quantitative evidence is provided to substantiate the choice of linearity and number of compartments. The only reference quoted [7] does not contain a detailed discussion on the subject, and from other literature this issue appears controversial $[8,9]$. Our observations suggest that, unlike the kinetics of IV injected insulin, those of SC insulin can be described by linear modelling (exponentials or compartments) only with great difficulty, if at all.

c) The reconstruction of SC absorption rate, given the IV insulin response and the plasma concentration after SC administration, is a classical 'inverse' problem, that is, the input is computed given the output and a model of the system. The techniques which are employed for its solution are usually referred to as deconvolution techniques. Deconvolution is a well-known ill-posed problem which cannot be solved crudely; precautions are usually taken, including, for instance, the explicit consideration of the whole time-course of the measured output (that is, plasma insulin after SC injection), typically using least-square estimators and the use of some stabilizing factors in order to prevent oscillatory solutions which are not related to the phenomena studied. Moreover, an estimate of the error of the reconstructed time course in each individual study should be provided. Fischer et al. [1] do not discuss these fundamental aspects. For instance, the absorption rate is computed by a simple deterministic inversion of the model equation in which only the concentrations in a narrow time interval are considered, instead of using the whole time course of plasma insulin concentration. The stability of such a method is known to be very poor. Some numbers are eventually obtained, but no measure of their reliability is given and due to the above considerations their accuracy seems rather unsatisfactory.

3. Due to the many built-in assumptions and computational difficulties discussed above, it is very difficult to judge the reliability of the figures provided in [1] on the rate of absorption of insulin. An independent validation would therefore have been highly advisable. For instance, the rate of appearance in the plasma of SC insulin would be simulated by a time-variable IV infusion and the rate predicted by the model compared with that used in the simulation.

4. The final comment concerns some aspects of data analysis and in particular the use of some indices computed from the data. Such indices are in general very useful but must have a conceptual rationale and should be appropriate to the particular problem. Otherwise they may be obscure in meaning and sometimes misleading. This is the case for the plasma glucose response area after insulin administration, used by Fischer et al. [1; Fig.2] as an index of the effect of insulin on glucose kinetics. This index is intrinsically saturable, even in the case of a completely linear glucose-insulin system, since the area enclosed by the glucose curve in a limited interval cannot increase indefinitely as the insulin dose increases. Thus, the saturation observed may re- 
flect a computational artefact, rather than a real phenomenon. A similar criticism applies to the authors' fitting of straight lines to the glucose decay curves, using different time intervals for different insulin doses. The validity of this method is questionable because a linear decay of glucose concentration is probably unphysiological and thus the parameters of the interpolated line have obscure meaning, if any at all.

In conclusion, two points should be re-emphasized: the first is that all the necessary modelling methods must be as rigorous and welldocumented as possible; the second is that when a model-based approach is proposed for an indirect measure, its inherent error must be known and judged acceptable for the intended purpose.

Yours sincerely,

C. Cobelli and A. Mari (Istituto di Elettrotecnica e di Elettronica) and E. Duner, F. Mollo and R. Nosadini (Istituto di Medicina Clinica, Università di Padova)

\section{References}

1. Fischer U, Freyse E-J, Jutzi E, Besch W, Raschke M, Höfler S, Albrecht $G$ (1983) Absorption rates of subcutaneously injected insulin in the dog as calculated from the plasma insulin levels by means of a simple mathematical model. Diabetologia 24: 196-201

2. Cobelli C, Mari A, Nosadini E, Del Prato S, Frigato A, Fabris R, Tiengo A, Crepaldi G, Berger A, Heding L (1983) Insulin kinetics following iv and sc injections in normals and Type I diabetics. Diabetes 32 (Suppl 1): 94A

3. Cobelli C, Mari A, Del Prato S, Duner E, Frigato A, Fabris R, Tiengo A, Crepaldi G, Berger A, Heding L (1983) Prolonged hypoglycaemic clamp technique to evaluate intravenous and subcutaneous kinetics of porcine and human insulin in normal subjects and Type 1 (insulin-dependent) diabetic patients. Diabetologia 25: 148 (Abstract)

4. Carson ER, Cobelli C, Finkelstein L (1983) The mathematical modeling of metabolic and endocrine systems. Model formulation, identification and validation. Wiley, New York

5. Sherwin RS, Kramer KJ, Tobin JD, Insel PA, Liljenquist JE, Berman M, Andres R (1974) A model of the kinetics of insulin in man. $\mathrm{J}$ Clin Invest 53:1481-1492

6. Tranberg KG, Dencker H (1978) Modeling of plasma disappearance of unlabeled insulin in man. Am J Physiol 235: E577-E585

7. Schlichtkrull J (1977) The absorption of insulin. Acta Paediatr Scand (Suppl) 270:97-102

8. Kobayashi T, Sawano S, Itoh T, Kosaka K, Hirayama H, Kasuya Y (1983) The pharmacokinetics of insulin after continuous subcutaneous infusion or bolus subcutaneous injection in diabetic patients. Diabetes 32: 331-336

9. Binder C (1969) Absorption of injected insulin. Acta Pharmacol Toxicol (Suppl 2) 27:1-84

Professor C. Cobelli

Istituto di Elettrotecnica e di Elettronica

Università di Padova

Via Gradenigo 6/A

I-35131 Padova, Italy

\section{Reply from the authors}

\section{Dear Sir}

With respect to the practicability of endocrine-metabolic models in vivo, one has to make a compromise between complexity and feasibility. We would like to comment only on those remarks by Cobelli et al. which refer to description of the appearance within the circulating plasma compartment of insulin injected $\mathrm{SC}$.
Most data published $[2,3,7,8,11]$ allow for the assumption that in the normoglycaemic and hyperglycaemic range - the metabolic clearance rate (MCR) of plasma insulin and the absorption of insulin injected SC do not in fact change in relation to blood glucose. During the study under discussion [3] no really hypoglycaemic levels were observed. It seems more important that, independent of the current blood glucose concentration, there are distinct differences in MCR between normal and diabetic individuals even if there are no anti-insulin antibodies in the latter. In control experiments employing IV insulin infusions $\left(3 \mathrm{mU} \cdot \mathrm{kg}^{-1} \cdot \mathrm{min}^{-1}\right)$ or injections $(50 \mathrm{mU} / \mathrm{kg})$, we found the following MCR of immunoreactive insulin (IRI): normal dogs $(n=15$, initial glycaemia $5.1 \pm 0.3 \mathrm{mmol} / 1), 27 \pm 2 \mathrm{ml} \cdot \mathrm{kg}^{-1}$. $\min ^{-1}$ (mean $\left.\pm \mathrm{SEM}\right)$; chronically diabetic dogs with normoglycaemia re-established by an artificial $\beta$ cell ( $n=7$, glycaemia at the beginning of the test $5.5 \pm 0.2 \mathrm{mmol} / 1), 43 \pm 6 \mathrm{ml} \cdot \mathrm{kg}^{-1} \cdot \mathrm{min}^{-1}$; the same dogs af ter nocturnal fasting and insulin withdrawal (initial glycaemia $17.0 \pm$ $3.6 \mathrm{mmol} / 1), 40 \pm 4 \mathrm{ml} \cdot \mathrm{kg}^{-1} \cdot \mathrm{min}^{-1}$. The differences in MCR between normal and diabetic animals were statistically significant $(p<0.01)$. We feel that in diabetic dogs as employed in our study [3] no bloodglucose dependence of MCR need be considered.

Correct conclusions on the bioavailability of a given insulin formulation cannot in fact be drawn from individual values but only from statistical means. This is confirmed by the following data, which were obtained in an earlier investigation in our laboratory [9]: the half time of plasma IRI after an IV bolus of $50 \mathrm{mU} / \mathrm{kg}$ in six normal Alsatian dogs was $2.53 \pm 0.34 \mathrm{~min}$. The intra-individual coefficient of variation was between $29 \%$ and $44 \%$ (mean $38 \%$ ) when eight tests were performed at intervals of $24 \mathrm{~h}$. In addition, MCR proved independent of the time of day but was obviously dependent on the initial plasma IRI level.

The linear one-compartment model was experimentally validated for describing plasma insulin kinetics after IV injection [9]. In 108 out of 120 tests in diabetic dogs ( 39 of them hyperglycaemic) both the linearized curve of $\log \left(\operatorname{IRI}_{\mathrm{t}}-\mathrm{IRI}_{\mathrm{O}}\right)$ versus time and the non-linear (monoexponential) least-squares regression analysis (Gauss-Newton gradient algorithm) of the measured values showed excellent approximation $(r=0.98)$. The remaining 12 test results yielded insufficient approximation by the use of these models. In general the goodness of fit was increased when a two-compartment model was employed. But in this case the accuracy of parameter estimates expressed as the percentage coefficient of variation always exceeded $100 \%$, which led us to conclude that any calculation of parameters would be worthless. Since other studies were likewise unable to show any advantages of higher-order models [11], the linear one-compartment model was used in the investigation under discussion [3] after experimental validation on other occasions [4]. During the editorial process we omitted these details and the respective discussions from the original manuscript to make it as short as possible. However, the full details on the reliability of our insulin measurements were included in the publication [3].

Different models might in fact be used to approximate the kinetics of insulin absorption after SC administration if different formulations (regular or long-acting insulin), different analytical methods (radioimmunoassay or scintillation counting after application of labelled insulin), or different techniques of administration (injection or infusion) are employed. The linear two-compartment model proved useful in our descriptive study (accuracy of fit expressed as percentage coefficient of uncertaintly was between $0.2 \%$ and $1.4 \%$ in the individual curves), which did not aim at simulating all the complex processes that may be assumed to take place when a certain amount of insulin is injected SC and a certain amount of insulin appears in the circulating pool. The parameters of such approximations can be used for the statistical characterization of a given insulin formulation. To obtain the parameters of such a low-order model, the transformation of related differential equations into a difference recursion formula is generally accepted, even if the estimation of performance indices (e.g. residual sum of squares, goodness of fit, Akaike information criterion) seems complicated [6]. In systems of a higher order, however, deconvolution techniques are to be used as performed by others in glucose or insulin models $[1,8,10]$.

The entire glucose-insulin system is in fact non-linear. However, in 
normal dogs we have been able to show linear input-output relations in the near-physiological range of glycaemia [5]. As mentioned above, no hypoglycaemic values occurred in our study on SC insulin absorption [3]. Thus a pure computational artefact counterfeiting saturation seems improbable indeed. Saturation of the insulin effect on many metabolic processes has been demonstrated in vivo and in vitro. Regardless of the explanation for the saturation kinetics of the effect of $\mathrm{SC}$ insulin on glycaemia in terms of mathematical modelling, their practical importance in the therapy of Type 1 (insulin-dependent) diabetes is evident. Thus it does not seem to us justified to disqualify the significance of measured values as 'questionable' or 'improbable" merely on the basis of theoretical conceptions.

In addition to the conclusions presented by Cobelli et al., we suggest that the practical applicability of models of the glucose-insulin system in vivo must not be disregarded if the connection between mathematical modeling and clinical or experimental practice is to be maintained.

Yours sincerely,

U. Fischer, E. Salzsieder, G. Albrecht and E. Jutzi

\section{References}

1. Cobelli C, Mari A (1983) Validation of mathematical models of complex endocrine-metabolic systems. A case study on a model of glucose regulation. Med Biol Eng Comput 21: 390-399

2. Ferrannini E, Wahren J, Faber O, Felig P, Binder C, DeFronzo RA (1983) Splanchnic and renal metabolism of insulin in human subjects: a dose-response study. Am J Physiol 244: E517-E527

3. Fischer U, Freyse EJ, Jutzi E, Besch W, Raschke M, Höfer S, Albrecht $G$ (1983) Absorption rates of subcutaneously injected insulin in the $\mathrm{dog}$ as calculated from the plasma insulin levels by means of a simple mathematical model. Diabetologia 24: 196-201
4. Fischer U, Jutzi E, Bombor H, Freyse EJ, Salzsieder E, Albrecht G, Besch W, Bruns W (1980) Assessment of an algorithm for the artificial $B$ cell using the normal insulin-glucose relationship in diabetic dogs and men. Diabetologia 18:97-107

5. Fischer U, Salzsieder E, Albrecht G, Jutzi E, Freyse EJ (1983) Can the control characteristics of the physiological glucose-insulin relationship be applied to an algorithm for closed loop insulin infusions in diabetes? In: Brunetti $\mathrm{P}$, Alberti KGMM, Albisser AM, Hepp KD, Massi Benedetti M (eds) Artificial systems for insulin delivery. Serono Symposia No.6. Raven Press, New York, pp 381-387

6. Isermann R (1977) Digitale Regelsysteme. Springer, Berlin Heidelberg New York, $\mathrm{p} 10$

7. Kobayashi T, Sawano S, Itoh T, Kosaka K, Hirayama H, Kasuya $Y(1983)$ The pharmacokinetics of insulin after continuous subcutaneous infusion or bolus subcutaneous injection in diabetic patients. Diabetes 32: 331-336

8. Pilo A, Ferrannini E, Navalesi R (1977) Measurement of glucoseinduced insulin delivery rate in man by deconvolution analysis. Am J Physiol 233: E500-E508

9. Röske D, Passow K (1979) Halbwertzeit und Verteilungsraum nicht markierten Insulins bei normalen und diabetischen Hunden: Einfluß auf den Algorithmus der Insulindosierung mittels einer künstlichen B-Zelle. Thesis for Medical Diploma, Ernst Moritz Arndt University, Greifswald, p1-38

10. Sherwin RS, Kramer KJ, Tobin JD, Insel PA, Liljenquist JE, Berman M, Andres R (1974) A model of the kinetics of insulin in man. $\mathrm{J}$ Clin Invest 53: 1481-1492

11. Tranberg KG, Dencker H (1978) Modelling of plasma disappearance of unlabelled insulin in man. Am J Physiol 235: E577-E585

Dr. Uwe Fischer

Central Institute of Diabetes 'Gerhardt Katsch'

DDR-2201 Karlsburg

GDR

\section{Book review}

Paediatric Immunology. J. F. Soothill, A. R. Hayward and C. B. S. Wood (eds) Edinburgh Boston Melbourne Oxford London: Blackwell Scientific Publications 1983. $490 \mathrm{pp}$, hardback, £35.00 ISBN 0632007249

The wide scope of present-day paediatric clinical immunology is outlined in the book by contributions from many leading authorities in this diverse field. The topic is dealt with comprehensively in three sections: Immunological mechanisms, General clinical immunology and Organ-based clinical immunology. The first section elucidates for the clinician mechanisms of adaptive immunity and allergic injury, complement and phagocyte function and the fundamentals of tissue types in relation to disease. Chapters on the development of immunity, the materno-fetal relationship in pregnancy and immunological aspects of infant feeding alert the paediatrician to his important role in the care of the newborn. A very informative account of specific immu- nodeficiency follows in Section 2, which also portrays current concepts regarding prophylactic immunisation, complement defects, defects of neutrophil function and management of the atopic child. Immunological aspects of diseases of the respiratory tract, gut, kidney, skin, blood and endocrine organs are illustrated in detail in the final section and there is a useful appendix giving normal ranges for various immunity function tests. An exellent book, clearly presented, it is well conceived to satisfy both the paediatrician floundering in the complexities (and complexes) of immunology and the clinical immunologist searching for up-date information in areas related to his own interests. The last chapter of the book, in particular, should interest readers of Diabetologia, since it highlights important recent advances in the immunogenetic aspects of diabetes. I recommend this book as a valuable asset to any medical library.

B. M. Dean (London) 